\title{
HEEGAARD FLOER HOMOLOGY OF SOME MAZUR TYPE MANIFOLDS
}

\author{
SELMAN AKBULUT AND ÇAĞRI KARAKURT
}

(Communicated by Daniel Ruberman)

\begin{abstract}
We show that an infinite family of contractible 4-manifolds has the same boundary as a special type of plumbing. Consequently the OzsváthSzabó invariants can be calculated algorithmically. We run this algorithm for the first few members of the family and list the resulting Heegaard Floer homologies. We also show that the rank of the Heegaard Floer homology can get arbitrarily large values in this family by using its relation with the Casson invariant. For comparison, we list the ranks of Floer homologies of all the examples of Brieskorn spheres that are known to bound contractible manifolds.
\end{abstract}

\section{MAZUR TYPE MANIFOLDS}

A 4-manifold is said to be Mazur type if it admits a handle decomposition consisting of a single handle at each index $\{0,1,2\}$ where the 2 -handle is attached in such a way that it cancels the 1-handle algebraically, 3. For $n \in \mathbb{N}$ and $k \in \mathbb{Z}$, let $W_{n}(k)$ be the Mazur type manifold whose handlebody picture is as drawn in Figure 1. The main purpose of this note is to calculate the Heegaard Floer homology group $\mathrm{HF}^{+}\left(\partial W_{n}(k)\right)$ for every $k$ and $n$. We are especially interested in the case where $k=0$, because the manifolds $W_{n}(0)$ are the corks which have been extensively used in the construction of exotic smooth structures on 4-manifolds, [5], [2]. Understanding the Floer homology of their boundary would be the first step in finding out how the smooth 4-manifold invariants change under surgery along these manifolds. The Heegaard Floer homology of another related family of contractible manifolds will be discussed in section 3 .

We first review some standard facts from [17, [18, and [19]. Heegaard Floer homology of any 3 -manifold $Y$ is a $\mathbb{Z} / 2 \mathbb{Z}$ graded abelian group which splits as a direct sum with respect to the $\operatorname{Spin}^{c}$ structures on $Y$. For any $\operatorname{Spin}^{c}$ structure $\mathfrak{t}$, the corresponding group $H F^{+}(Y, \mathfrak{t})$ admits an endomorphism $U$ which preserves the $\mathbb{Z} / 2 \mathbb{Z}$ grading. This endomorphism equips Heegaard Floer homology with the structure of a $\mathbb{Z}[U]$ module. When a $\operatorname{Spin}^{c}$ structure is torsion, the associated component of Heegaard Floer homology admits an absolute $\mathbb{Q}$-grading. In this case $U$ has degree -2 . When $Y$ is an integral homology sphere, there is a single

Received by the editors April 28, 2012 and, in revised form, September 13, 2012 and December 3, 2012.

2010 Mathematics Subject Classification. Primary 57R58, 57R65, 57R57.

The first named author is partially supported by NSF FRG grants DMS-1065879 and DMS0905917.

The second named author is supported by a Simons fellowship and NSF FRG grant DMS1065718 . 


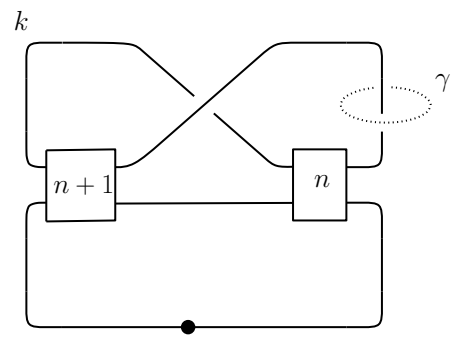

Figure 1. The Mazur type manifold $W_{n}(k)$.

Spin $^{c}$ component where the absolute grading in fact takes integer values. In this case the parity of this $\mathbb{Z}$-grading agrees with the $\mathbb{Z} / 2 \mathbb{Z}$ grading, and the following splitting occurs:

$$
H F^{+}(Y)=\mathcal{T}_{(d)}^{+} \oplus H F^{\mathrm{red}}(Y)
$$

where $H F^{\text {red }}(Y)$ is a finitely generated abelian group, and $\mathcal{T}_{(d)}^{+}$is a copy of the module $\mathbb{Z}\left[U, U^{-1}\right] / U \mathbb{Z}[U]$ graded so that the minimal degree element has degree d. This minimal degree is called the correction term of $Y$. Moreover, the Euler characteristic of the reduced part satisfies

$$
\chi\left(H F^{\mathrm{red}}(Y)\right)=\lambda(Y)+d(Y) / 2
$$

where $\lambda(Y)$ is the Casson invariant of $Y, 4$.

It is evident from the definition that any Mazur type manifold that $W$ is contractible, so its boundary is an integral homology sphere. Therefore $\mathrm{HF}^{+}(\partial W)$ has the structure mentioned above. In addition to that, we have the following restrictions.

Proposition 1.1. Suppose that an integral homology sphere bounds a contractible manifold. Then its correction term is zero and its Casson invariant is even.

Proof. The correction term of a homology sphere is invariant under $\operatorname{Spin}^{c}$ rational homology cobordisms. Any contractible manifold $W$ is an integral homology ball. We can also regard it as an integral homology cobordism from $S^{3}$ to $\partial W$, by removing a small ball from it. Since the correction term of the 3 -sphere is zero, we have $d(\partial W)=0$. The second statement is a consequence of the fact that the Mod 2 reduction of the Casson invariant of any integral homology sphere is in fact the Rohlin invariant, which can be computed from the signature of the Spin 4-manifold it bounds. Since the signature of any contractible manifold is zero, the second part follows.

Next we prove that up to a grading change, the Heegaard Floer homology is independent of the framing of the 2-handle of a Mazur type manifold. See [23], for an analogous statement in Instanton Floer homology.

Proposition 1.2. Let $W(k)$ be a Mazur type manifold, $k$ being the framing of the 2-handle. Then for any $k, k^{\prime} \in \mathbb{Z}$, there is a $U$ equivariant isomorphism between $H F^{+}(\partial W(k))$ and $\mathrm{HF}^{+}\left(\partial W\left(k^{\prime}\right)\right)$ which preserves $\mathbb{Z} / 2 \mathbb{Z}$ gradings. Moreover, this isomorphism also preserves absolute $\mathbb{Q}$ gradings if the meridional circle $\gamma$ of the 2 -handle bounds a genus one Seifert surface in $\partial W(k)$ for every $k$. 
Proof. By Equation (1.1) and Proposition 1.1 it suffices to show that $H F^{\mathrm{red}}(W(k))$ is independent of $k$. To see this, we use the surgery exact triangle [18. Observe that -1 framed surgery on the curve $\gamma$ inside $\partial W(k)$ gives $\partial W(k+1)$ and 0 surgery along the same curve gives $S^{1} \times S^{2}$. The Heegaard Floer homology of $S^{1} \times S^{2}$, which is supported in the torsion $\operatorname{Spin}^{c}$ structure $\mathfrak{t}_{0}$, is given by $H F^{+}\left(S^{1} \times S^{2}, \mathfrak{t}_{0}\right)=$ $\mathcal{T}_{(1 / 2)} \oplus \mathcal{T}_{(-1 / 2)}$. The surgery exact triangle can be read as follows:

$$
\begin{aligned}
& \cdots \stackrel{f_{3}}{\longrightarrow} H F_{p}^{+}(\partial W(k+1)) \stackrel{f_{1}}{\longrightarrow} H F_{p-\frac{1}{2}}^{+}\left(S^{1} \times S^{2}, \mathfrak{t}_{0}\right) \stackrel{f_{2}}{\longrightarrow} \\
& \stackrel{f_{2}}{\longrightarrow} H F_{p-1}^{+}(\partial W(k)) \stackrel{f_{3}}{\longrightarrow} \cdots
\end{aligned}
$$

The homomorphisms $f_{1}$ and $f_{2}$ are homogeneous of degree $-1 / 2$. To see this recall that each one of $f_{1}, f_{2}$, and $f_{3}$ is induced from a 4 -dimensional cobordism. For example, $f_{1}$ corresponds to a 2-handle attachment along $\gamma$ with framing 0 giving a cobordism $C$ with boundary $\partial C=-\partial W(k+1) \sqcup S^{1} \times S^{2}$. Now, every Spin ${ }^{c}$ structure on $S^{1} \times S^{2}$ has a unique extension on $C$. Therefore $f_{1}= \pm F_{C, \mathfrak{s}_{0}}$ where $\mathfrak{s}_{0}$ is the torsion $\operatorname{Spin}^{c}$ structure on $C$, and $F_{C, \mathfrak{F}_{0}}$ is the homomorphism induced by the pair $\left(C, \mathfrak{s}_{0}\right)$. Then the degree of $f_{1}$ is given by $\left(c_{1}\left(\mathfrak{s}_{0}\right)^{2}-3 \sigma(C)-2 \chi(C)\right) / 4=-1 / 2$. That $f_{2}$ is homogeneous of degree $-1 / 2$ can be proven similarly.

Next we analyze the exact sequence (1.2). When $p$ is large there is no contribution coming from $H F^{\mathrm{red}}$, so all the groups appearing in the sequence (1.2) are isomorphic to either $\mathbb{Z}$ or 0 . When $p$ is large and even $f_{2}=0$, so $f_{1}$ is an isomorphism. Similarly, when $p$ is large and odd $f_{2}$ is an isomorphism. By $U$-equivariance, $f_{1}$ sends $\mathcal{T}_{(0)}^{+} \subset H F^{+}(\partial W(k+1))$ isomorphically to $\mathcal{T}_{(-1 / 2)}^{+} \subset H F^{+}\left(S^{1} \times S^{2}\right)$. Similarly, $f_{2}$ induces an isomorphism between

$$
\mathcal{T}_{(1 / 2)}^{+} \subset H F^{+}\left(S^{1} \times S^{2}\right)
$$

and

$$
\mathcal{T}_{(0)}^{+} \subset H F^{+}(\partial W(k)) \text {. }
$$

Hence we have $\operatorname{coker}\left(f_{2}\right) \simeq H F^{\mathrm{red}}(W(k))$ and $\operatorname{ker}\left(f_{1}\right) \simeq H F^{\mathrm{red}}(W(k+1))$. By exactness, $f_{3}$ induces an isomorphism between $H F^{\mathrm{red}}(W(k))$ and $H F^{\mathrm{red}}(W(k+1))$.

Unlike the other two homomorphisms appearing in the exact triangle, $f_{3}$ may not be homogeneous. Even so, we claim that it preserves the $\mathbb{Z} / 2 \mathbb{Z}$ grading. When we attach a 2-handle along $\gamma \subset \partial W(k)$ with framing -1 and call the resulting cobor$\operatorname{dism} X(k)$, the Seifert surface of $\gamma$ gives rise to a closed surface $\Sigma \subset X(k)$ whose homology class generates $H_{2}(X(k), \mathbb{Z}) \simeq \mathbb{Z}$. Let $\mathfrak{s}_{r}, r \in \mathbb{Z}$, be the Spin ${ }^{c}$ structure on $X(k)$ with $\left\langle c_{1}\left(\mathfrak{s}_{r}\right),[\Sigma]\right\rangle=2 r+1$. Denote by $F_{X(k), \mathfrak{s}_{r}}^{+}$, the homomorphism associated to the $\operatorname{Spin}^{c}$ structure $\mathfrak{s}_{r}$. It is known that

$$
f_{3}=\sum_{r \in \mathbb{Z}} \pm F_{X(k), \mathfrak{s}_{r}}^{+}
$$

for some choice of signs. Each $F_{X(k), \mathfrak{s}_{r}}^{+}$is homogeneous of degree

$$
\left(c_{1}\left(\mathfrak{s}_{r}\right)^{2}-3 \sigma(X(k))-2 \chi(X(k))\right) / 4=-r(r+1)
$$

which is even for every $r$. 
For the last part, suppose the genus of $\Sigma$ is one. Then the $\operatorname{Spin}^{c}$ structure $\mathfrak{s}_{r}$ satisfies

$$
\left|\left\langle c_{1}\left(\mathfrak{s}_{r}\right),[\Sigma]\right\rangle\right|+[\Sigma] \cdot[\Sigma]>2 g-2
$$

for every $r \in \mathbb{Z}-\{-1,0\}$, so it admits a non-trivial adjunction relation, 21]. Hence by Equation (1.3), $f_{3}$ can be written as

$$
f_{3}=\phi+U \cdot \psi
$$

for some $\phi$ and $\psi$, where $\phi$ is the portion of $f_{3}$ coming from $\mathfrak{s}_{-1}$ and $\mathfrak{s}_{0}$. The degree calculation above shows that $\phi$ is homogeneous of degree 0 . We will show that $\phi$ restricts to an isomorphism between $H F^{\mathrm{red}}(\partial W(k))$ and $H F^{\mathrm{red}}(\partial W(k+1))$. Both of these groups are filtered as follows:

$$
\operatorname{ker}(U) \subseteq \operatorname{ker}\left(U^{2}\right) \subseteq \cdots \subseteq \operatorname{ker}\left(U^{s}\right)=H F^{\mathrm{red}} .
$$

The isomorphism $f_{3}$ respects this filtration. In particular it induces an isomorphism on $\operatorname{ker}\left(U^{t}\right) / \operatorname{ker}\left(U^{t-1}\right)$ for all $t=1,2, \cdots, s$. By Equation (1.4), the restriction of $f_{3}$ on each of these groups is the same as $\phi$. Having seen that $\phi$ is an isomorphism on each $\operatorname{ker}\left(U^{t}\right) / \operatorname{ker}\left(U^{t-1}\right)$, we conclude that $\phi$ is an isomorphism on the whole $H F^{\text {red }}(\partial W(k))$.

Now we focus our attention on the family of Mazur type manifolds $W_{n}(k)$ of Figure 1. In view of the previous proposition, one would like to have a genus one Seifert surface of the curve $\gamma \subset \partial W_{n}(k)$ for every $n$ and $k$. One can construct such a Seifert surface easily for $n=1$ :

Proposition 1.3. The curve $\gamma$ in Figure 1 bounds a surface of genus one in $\partial W_{1}(k)$.

Proof. Observe that $W_{1}(k)$ is the same as the manifold $W^{-}(0, k)$ of [3]. We draw a surgery picture of the boundary of this manifold in Figure 2 after replacing the circle with a dot and with a 0 -framed 2 -handle. We slide $\gamma$ over the 0 -framed circle. Now the disk bounded by $\gamma$ intersects the $k$-framed circle twice with opposite signs. Therefore we can puncture the disk at these points and add a tube parallel to a portion of the circle. The resulting genus 1 surface bounded by $\gamma$ is the required Seifert surface.

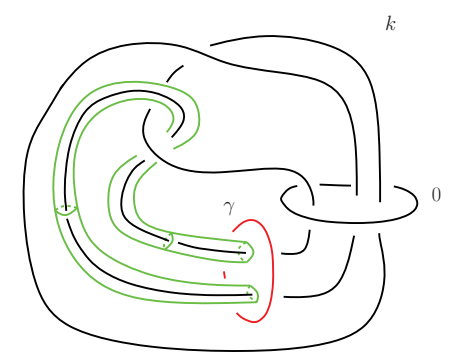

FiguRE 2. Seifert surface of $\gamma$ in $\partial W_{1}(k)$

Remark 1.1. Combining Propositions 1.2 and 1.3, we see that $W_{1}(k)$ is an infinite family of integral homology spheres with isomorphic Heegaard Floer homology. It was proven in [3] that one member of this family has boundary the Brieskorn sphere 
$\Sigma(2,5,7)$ (also two other members have boundaries $\Sigma(3,4,5)$, and $\Sigma(2,3,13)$ ). One can incorporate the combinatorial description of Heegaard Floer homology for Brieskorn spheres [13, 22] (or see Section 2) to conclude

$$
H F^{+}\left(-\partial W_{1}(0)\right) \simeq H F^{+}(-\Sigma(2,5,7)) \simeq \mathcal{T}_{(0)}^{+} \oplus \mathbb{Z}_{(0)} \oplus \mathbb{Z}_{(0)} .
$$

This was previously observed in [1] by similar techniques, but applied in a somewhat different way.

In our next result we show that the homology sphere $\partial W_{n}(2 n+1)$ bounds a special type of plumbing, generalizing a result of [3]. After the completion of the first draft this paper, it was brought to our attention that the same result was also proven by Maruyama [11.

Theorem 1.4 (11]). For all $n=1,2,3, \ldots$, the 3 -manifold $\partial W_{n}(2 n+1)$ bounds a 4-manifold which is obtained by taking several disk bundles over spheres and plumbing them together according to the almost rational graph given in Figure 3 ,

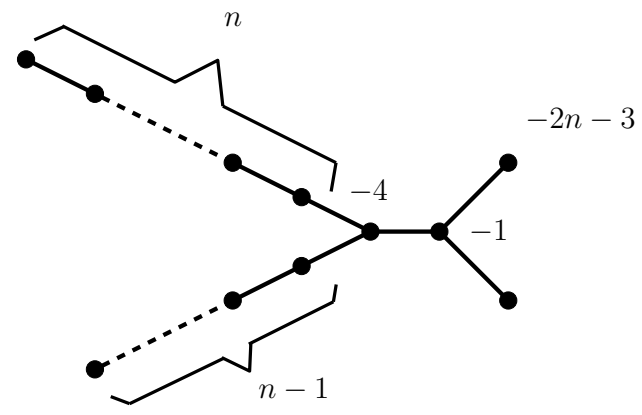

Figure 3. The plumbing configuration bounded by $\partial W_{n}(2 n+1)$. Unlabeled vertices have weight -2 .

Proof. The proof follows by a sequence of handlebody moves. They are indicated in Figure 4 for $n=3$. The general case can be handled similarly.

For any oriented 3-manifold $Y$, let $-Y$ denote the the same 3-manifold with the opposite orientation. It is easy to determine the effect of orientation reversal on Heegaard Floer homology, [18, [19. In view of this fact, our theorem implies that the Heegaard Floer homology of the family of 3-manifolds that we are interested in can be calculated combinatorially.

Corollary 1.5. There is an algorithm calculating $\mathrm{HF}^{+}\left(-\partial W_{n}(2 n+1)\right)$ for all $n$.

Proof. Follows from the result of Nemethi [13. (see also, Ozsváth and Szabó [22]). The only thing that we should check is whether the plumbing graph $G$ is almost rational in the sense of 13 . Definition 8.1. Indeed by replacing the weight of the central vertex from -1 to -3 we get a rational graph (see section 6.2 of [13]).

We write a computer code in Magma that implements Nemethi's algorithm to calculate the Heegaard Floer homology of $-\partial W_{n}(2 n+1)$. We list the Heegaard Floer homologies of the first three members of this family in Corollary 1.6 to give the reader an idea of what these groups look like. See Table 1 for the data that 


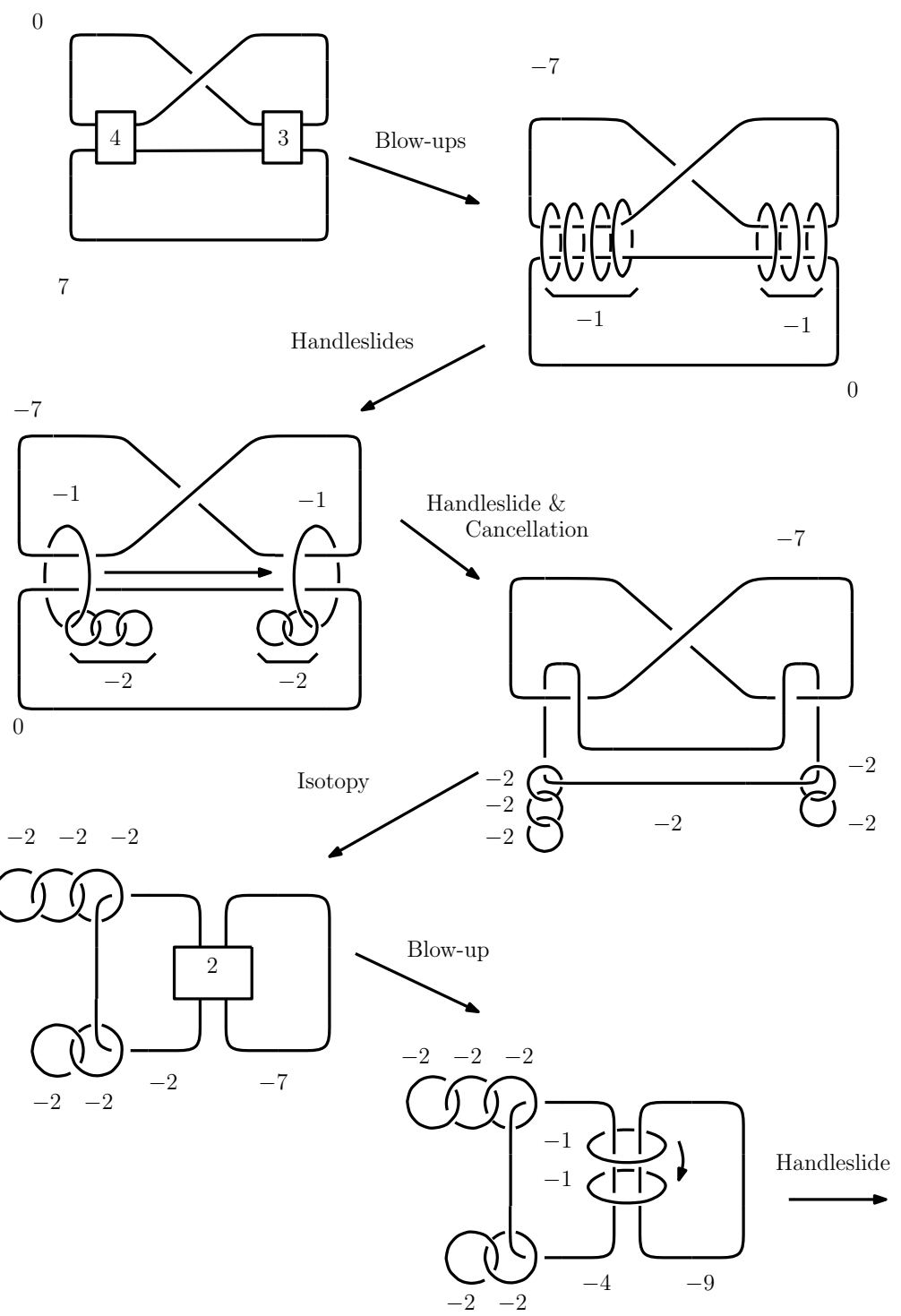

FiguRE 4. Handlebody moves showing that $\partial W_{n}(2 n+1)$ bounds the plumbing indicated in Figure 3 .

determines $\mathrm{HF}^{+}\left(-\partial W_{n}(2 n+1)\right.$ for $n=1, \ldots, 7$. Our code is available online at [9].

Corollary 1.6. Let $\mathbb{Z}_{(d)}^{r}$ be the quotient module $\mathbb{Z}[U] / U^{r} \mathbb{Z}[U]$ graded so that $U^{r-1}$ has degree $d$. Then we have the following identifications:

(1) $H F^{+}\left(-W_{1}(3)\right) \simeq \mathcal{T}_{0}^{+} \bigoplus\left(\mathbb{Z}_{(0)}\right)^{2}$,

(2) $H F^{+}\left(-W_{2}(5)\right) \simeq \mathcal{T}_{0}^{+} \oplus\left(\mathbb{Z}_{(0)}\right)^{4} \oplus\left(\mathbb{Z}_{(2)}\right)^{2} \oplus\left(\mathbb{Z}_{(10)}\right)^{2}$

(3) $H F^{+}\left(-W_{3}(7)\right) \simeq \mathcal{T}_{0}^{+} \oplus\left(\mathbb{Z}_{(0)}\right)^{4} \oplus\left(\mathbb{Z}_{(0)}^{2}\right)^{2} \oplus\left(\mathbb{Z}_{(2)}\right)^{2} \oplus\left(\mathbb{Z}_{(4)}\right)^{2} \oplus\left(\mathbb{Z}_{(12)}\right)^{2} \oplus$ $\left(\mathbb{Z}_{(14)}\right)^{2} \oplus\left(\mathbb{Z}_{(18)}\right)^{2} \oplus\left(\mathbb{Z}_{(42)}\right)^{2}$. 
Presumably, it is possible to find a closed formula for these Heegaard Floer homologies for every $n$ using Nemethi's techniques. Though the authors were not able to find such a formula, one can easily verify that the rank of Heegaard Floer homology gets arbitrarily large with $n$ using a different method.

Corollary 1.7. $\operatorname{rank} H F^{\mathrm{red}}\left(-\partial W_{n}(k)\right)=-\lambda\left(\partial W_{n}(k)\right)=n(n+1)(n+2) / 3$.

Proof. When $Y$ bounds an almost rational graph, $H F^{+}(-Y)$ is supported only in even degrees $\left[22\right.$, implying $\chi\left(H F^{\mathrm{red}}(-Y)\right)=\operatorname{rank} H F^{\mathrm{red}}(-Y)$. Therefore by Theorem 1.4 Proposition 1.2 and Proposition 1.1, it suffices to show that $\lambda\left(-W_{n}(2 n+1)\right)=n(n+1)(n+2) / 3$. This calculation is done in 8]. Alternatively, this can be seen from the Casson invariant formula for plumbings given in section 2.4.2 of 13 . Note that the formula involves calculating certain minors of the intersection matrix whose rank increases with $n$. One can get around that problem by realizing that each minor actually corresponds to the order of the first homology of the 3-manifold obtained by deleting a vertex, and converting the resulting integral surgery diagram to a rational surgery diagram. The details are left to the reader (see the discussion in Section 5.1 of [15]).

Remark 1.2. It is known that every even integer can be realized as the Casson invariant of some homology sphere bounding a Mazur type manifold [10]. However it is still an open question whether this could be achieved in the subclass of integral homology spheres which bound both a Mazur type manifold and an almost rational plumbing.

\section{USER'S GUIDE TO NEMETHI'S ALGORIThM}

The aim of this section is to summarize the method of calculating the Heegaard Floer homology groups given in [13]. This procedure is indicated schematically in Figure 5. In order to get the Heegaard Floer homology, one needs to calculate some intermediate objects, so-called computation sequence, tau function and graded root. We will define these objects and tell how they are used in the process in the following subsections. We are going to discuss only a special case where the 3manifold is an integral homology sphere, though the algorithm more generally works for rational homology spheres as well if one has an extra ingredient associated to Spin ${ }^{c}$ structures.

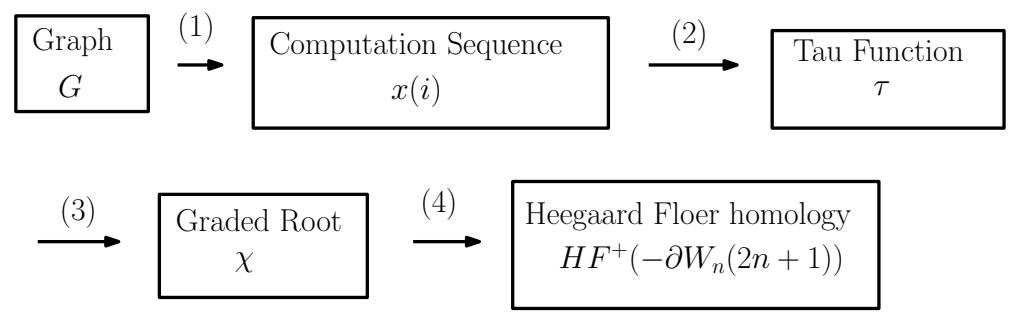

FiguRE 5. Steps of Nemethi's method 
2.1. From graph to computation sequence: Let $G_{n}$ be the plumbing graph indicated in Figure 3. Denote its vertices by $\left\{v_{0}, v_{1}, \cdots, v_{2 n+2}\right\}$. Choose the indices so that $v_{0}$ is the vertex with decoration -1 . For any vertex $v$, let $m(v)$ and $\delta(v)$ denote the weight of $v$ and the number of edges connected to $v$ respectively. We distinguish the vertex $v_{0}$ because it is the only one satisfying the inequality $|m(v)|<$ $\delta(v)$. Let $X\left(G_{n}\right)$ be the plumbed 4-manifold. Each vertex $v$ represents a generator of $H_{2}\left(X\left(G_{n}\right)\right)$ which will be denoted by the same letter. Let $($,$) be the intersection$ form on $H_{2}\left(X\left(G_{n}\right)\right)$. Then

$$
\left(v_{i}, v_{j}\right)=\left\{\begin{array}{l}
m\left(v_{i}\right) \text { if } i=j \\
1 \text { if there is an edge connecting } v_{i} \text { to } v_{j} \\
0 \text { if there is no edge connecting } v_{i} \text { to } v_{j} .
\end{array}\right.
$$

Inductively define a sequence $x(i) \in H_{2}\left(X\left(G_{n}\right)\right)$ by first requiring that $x(0)=$ 0 , and then obtaining $x(i+1)$ from $x(i)$ by connecting them via a computation sequence $y_{1}, y_{2}, \cdots, y_{l}$ which is defined as follows:

(1) $y_{1}=x(i)+v_{0}$.

(2) Suppose $y_{r}$ is known. If $\left(y_{r}, v_{j}\right)>0$ for some $j \neq 0$ then define $y_{r+1}:=$ $y_{r}+v_{j}$.

(3) Repeat step (2) until you find $y_{l}$, such that $\left(y_{l}, v_{j}\right) \leq 0$ for all $j \neq 0$, then declare that $x(i+1):=y_{l}$.

Although $x(i)$ is an infinite sequence, only a finite portion of it will be used in the next stage of the algorithm.

2.2. From computation sequence to tau function: Using the output of the previous step, we inductively construct a function $\tau: \mathbb{N} \rightarrow \mathbb{Z}$ :

- $\tau(0)=0$.

- $\tau(i+1)=\tau(i)+1-\left(x(i), v_{0}\right)$.

It can be shown that $\tau$ is ultimately increasing. Indeed if $i_{0}$ is the first index with $\tau\left(i_{0}\right)=2$, then $\tau(i) \leq \tau(i+1)$ for all $i \geq i_{0}$. For the remaining part, we only need the restriction of $\tau$ to the set $\left\{1,2,3, \cdots, i_{0}\right\}$.

Regard $\tau$ as a finite sequence. Choose a subsequence by first killing all the consecutive repetitions, and then taking all the local maxima and local minima of what is left. We also drop the last entry which is equal to 2 . For example, the tau function obtained from the graph $G_{1}$ is $[0,1,0,0,0,0,0,0,0,0,0,1,0,0,0,1,1,1,1,1,1,2]$ which reduces to $[0,1,0,1,0]$ after the reduction. We list the reduced tau functions of $G_{n}$ for $n=1, \ldots, 7$ in Table 1 .

2.3. From tau function to graded root: Given a natural number $i$, let $R_{i}$ denote the infinite tree with the vertex set $\mathcal{V}:=\left\{v^{j}: j \geq i\right\}$ and the edge set $\mathcal{E}:=\left\{\left[v^{j}, v^{j+1}\right]: j \geq i\right\}$. This tree also admits a grading function $\chi: \mathcal{V} \rightarrow \mathbb{Z}$, with $\chi\left(v^{j}\right)=j$. From the (reduced) tau function $\tau:\{0,1,2, \cdots, 2 k\}$, we can construct an infinite tree

$$
R_{\tau}:=R_{\tau(0)} \sqcup R_{\tau(1)} \sqcup R_{\tau(2)} \cdots \sqcup R_{\tau(2 k)} / \sim,
$$

where the equivalence relation $\sim$ is defined as follows: the reduced $\tau$ function attains its local maxima at odd integers. Therefore $R_{\tau(2 i+1)}$ naturally injects into both $R_{\tau(2 i)}$, and $R_{\tau(2 i+2)}$ in a way that preserves the grading. We identify $R_{\tau(2 i+1)}$ with its images in $R_{\tau(2 i)}$, and $R_{\tau(2 i+2)}$ for every $i=0,1,2, \ldots, k-1$. Then the grading functions on each $R_{\tau(j)}$ descend to a grading function $\chi_{\tau}$ on $R_{\tau}$. The pair $\left(R_{\tau}, \chi_{\tau}\right)$ is called the graded root associated with $\tau$. An illustration of this 


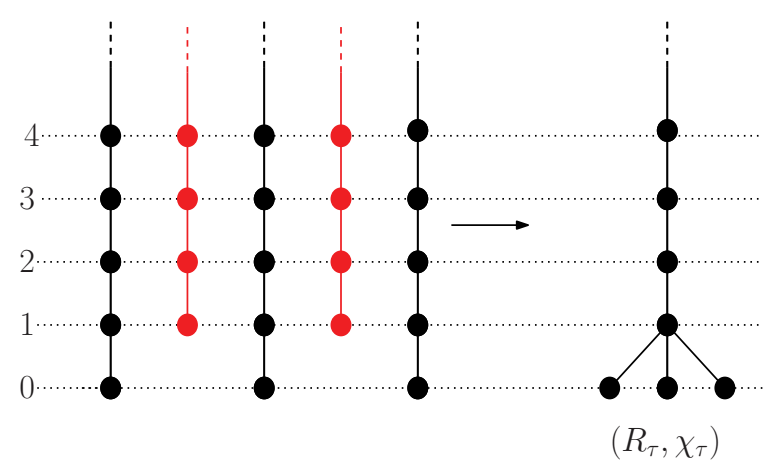

Figure 6. Graded Root of $G_{1}$.

construction is given in Figure [6 when $\tau=[0,1,0,1,0]$. We draw the graded roots of the graphs $G_{n}$, for $n=1,2,3$ in Figure 7

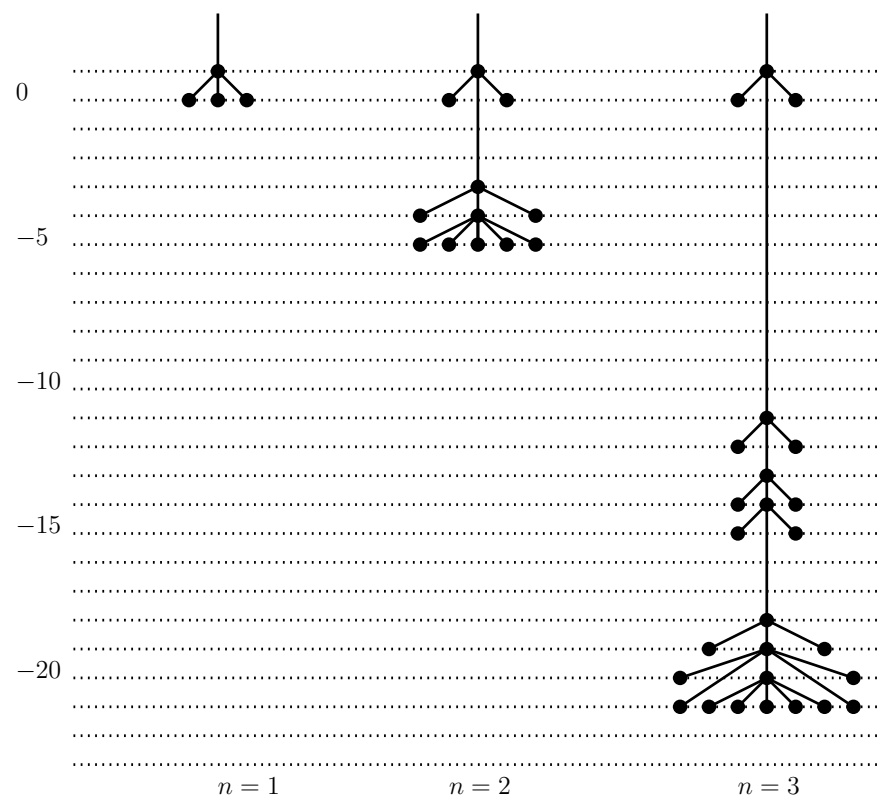

Figure 7 . The graded roots generated by $\tau$ functions in Table 1 , for $n=1,2,3$.

2.4. From graded root to Heegaard-Floer Homology: Let $\mathcal{V}$ and $\mathcal{E}$ denote the vertex and edge sets of $R_{\tau}$. Define

$$
\begin{aligned}
\mathbb{H}\left(R_{\tau}, \chi_{\tau}\right)=\left\{\phi: \mathcal{V} \rightarrow \mathcal{T}_{(0)}^{+}: U \cdot \phi(v)\right. & =\phi(w) \\
& \text { whenever } \left.[v, w] \in \mathcal{E} \text { and } \chi_{\tau}(v)<\chi_{\tau}(w)\right\}
\end{aligned}
$$

The set $\mathbb{H}\left(R_{\tau}, \chi_{\tau}\right)$ naturally admits the structure of a $\mathbb{Z}[U]$-module. One can define a grading on it as follows: $\phi \in \mathbb{H}\left(R_{\tau}, \chi_{\tau}\right)$ is said to be homogeneous of degree $d$ if $\phi(v) \in \mathcal{T}_{(0)}^{+}$is homogeneous of degree $d-2 \chi_{\tau}(v)$ for every $v \in \mathcal{V}$. Finally, the 
Heegaard Floer homology is isomorphic to $\mathbb{H}\left(R_{\tau}, \chi_{\tau}\right)$ with a grade shift. In the cases of interest, the exact amount of grade shift can be found by requiring that $d\left(-\partial W_{n}(2 n+1)\right)=0$.

\section{SOME BRIESKORN SPHERES BOUNDING CONTRACTIBLE MANIFOLDS}

Thus far we have observed that if a Mazur type manifold has the same boundary as a plumbing up to change of framings, then its Ozsváth-Szabó invariant can be calculated combinatorially. We have seen this happening in an infinite family of contractible manifolds. In [7, Casson and Harer constructed many examples of this sort. Our goal in this section is to study Heegaard Floer homologies of their examples, and to make a comparison with the results of the calculations that we made in the previous sections.

TABLE 1. Reduced $\tau$ functions associated to the plumbings indicated in Figure 3 for $n=1,2, \ldots, 7$.

\begin{tabular}{|c|c|}
\hline$n=1$ & {$[0,1,0,1,0]$} \\
\hline$n=2$ & {$[0,1,-4,-3,-5,-4,-5,-4,-5,-4,-5,-4,-5,-3,-4,1,0]$} \\
\hline$n=3$ & $\begin{array}{l}{[0,1,-12,-11,-14,-13,-15,-14,-19,-18,-20,-19,-21} \\
-19,-21,-20,-21,-20,-21,-20,-21,-20,-21,-19,-21 \\
-19,-20,-18,-19,-14,-15,-13,-14,-11,-12,1,0]\end{array}$ \\
\hline$n=4$ & $\begin{array}{l}{[0,1,-24,-23,-27,-26,-29,-28,-41,-40,-43,-42,-45} \\
-43,-46,-45,-47,-46,-51,-50,-52,-51,-53,-51,-54,-52, \\
-54,-52,-54,-53,-54,-53,-54,-53,-54,-53,-54,-52,-54, \\
-52,-54,-51,-53,-51,-52,-50,-51,-46,-47,-45,-46,-43, \\
-45,-42,-43,-40,-41,-28,-29,-26,-27,-23,-24,1,0]\end{array}$ \\
\hline$n=5$ & $\begin{array}{l}{[0,1,-40,-39,-44,-43,-47,-46,-71,-70,-74,-73,-77,-75,} \\
-79,-78,-81,-80,-93,-92,-95,-94,-97,-95,-99,-97,-100,-98 \\
-101,-100,-102,-101,-106,-105,-107,-106,-108,-106,-109,-107, \\
-110,-107,-110,-108,-110,-108,-110,-109,-110,-109,-110,-109 \\
-110,-109,-110,-108,-110,-108,-110,-107,-110,-107,-109,-106, \\
-108,-106,-107,-105,-106,-101,-102,-100,-101,-98,-100,-97,-99, \\
-95,-97,-94,-95,-92,-93,-80,-81,-78,-79,-75,-77,-73,-74,-70 \\
-71,-46,-47,-43,-44,-39,-40,1,0]\end{array}$ \\
\hline$n=6$ & $\begin{array}{l}{[0,1,-60,-59,-65,-64,-69,-68,-109,-108,-113,-112,-117,-115,-120,} \\
-119,-123,-122,-147,-146,-150,-149,-153,-151,-156,-154,-158,-156, \\
-160,-159,-162,-161,-174,-173,-176,-175,-178,-176,-180,-178,-182, \\
-179,-183,-181,-184,-182,-185,-184,-186,-185,-190,-189,-191,-190, \\
-192,-190,-193,-191,-194,-191,-195,-192,-195,-192,-195,-193,-195, \\
-193,-195,-194,-195,-194,-195,-194,-195,-194,-195,-193,-195,-193, \\
-195,-192,-195,-192,-195,-191,-194,-191,-193,-190,-192,-190,-191, \\
-189,-190,-185,-186,-184,-185,-182,-184,-181,-183,-179,-182,-178, \\
-180,-176,-178,-175,-176,-173,-174,-161,-162,-159,-160,-156,-158, \\
-154,-156,-151,-153,-149,-150,-146,-147,-122,-123,-119,-120,-115, \\
-117,-112,-113,-108,-109,-68,-69,-64,-65,-59,-60,1,0]\end{array}$ \\
\hline$n=7$ & $\begin{array}{l}{[0,1,-84,-83,-90,-89,-95,-94,-155,-154,-160,-159,-165,-163,-169,} \\
-168,-173,-172,-213,-212,-217,-216,-221,-219,-225,-223,-228,-226, \\
-231,-230,-234,-233,-258,-257,-261,-260,-264,-262,-267,-265,-270, \\
-267,-272,-270,-274,-272,-276,-275,-278,-277,-290,-289,-292,-291, \\
-294,-292,-296,-294,-298,-295,-300,-297,-301,-298,-302,-300,-303, \\
-301,-304,-303,-305,-304,-309,-308,-310,-309,-311,-309,-312,-310, \\
-313,-310,-314,-311,-315,-311,-315,-312,-315,-312,-315,-313,-315, \\
-313,-315,-314,-315,-314,-315,-314,-315,-314,-315,-313,-315,-313, \\
-315,-312,-315,-312,-315,-311,-315,-311,-314,-310,-313,-310,-312, \\
-309,-311,-309,-310,-308,-309,-304,-305,-303,-304,-301,-303,-300, \\
-302,-298,-301,-297,-300,-295,-298,-294,-296,-292,-294,-291,-292, \\
-289,-290,-277,-278,-275,-276,-272,-274,-270,-272,-267,-270,-265, \\
-267,-262,-264,-260,-261,-257,-258,-233,-234,-230,-231,-226,-228, \\
-223,-225,-219,-221,-216,-217,-212,-213,-172,-173,-168,-169,-163, \\
-165,-159,-160,-154,-155,-94,-95,-89,-90,-83,-84,1,0]\end{array}$ \\
\hline
\end{tabular}


Given pairwise relatively prime natural numbers $p, q, r$, the Brieskorn manifold $\Sigma(p, q, r)$ is defined as the intersection of the complex hypersurface $z_{1}^{p}+z_{2}^{q}+z_{3}^{r}=0$ with a 5-dimensional sphere centered at the origin with small radius.

Proposition 3.1 ([7]). The following Brieskorn manifolds bound Mazur type manifolds.

(1) $\Sigma(p, p s \pm 1, p s \pm 2), p$ odd.

(2) $\Sigma(p, p s-1, p s+1)$, p even, $s$ odd.

Most of these Mazur type manifolds do not give us corks even if we are allowed to change the framing of the 2-handle. This is because the two handles are knotted, so there is no obvious involution on the boundary. However, there is one particular subfamily found by Maruyama which is closely related to the corks we are interested in.

Proposition $3.2([12])$. The Brieskorn manifold $\Sigma(2 n+1,2 n+2,2 n+3)$ bounds the manifold $W_{n}(2 n+2)$.

Any Brieskorn manifold bounds an almost rational plumbing. Indeed, let $e_{0}, p^{\prime}$, $q^{\prime}, r^{\prime}$ be the unique integers satisfying $0<p^{\prime}<p, 0<q^{\prime}<q, 0<r^{\prime}<r$, and

$$
e_{0} p q r+p^{\prime} q r+p q^{\prime} r+p q r^{\prime}=-1 .
$$

Then $\Sigma(p, q, r)$ is the Seifert fibered space with Seifert invariants $\left(e_{0}, p, p^{\prime}, q, q^{\prime}, r, r^{\prime}\right)$, and as such it bounds a star shaped plumbing with three branches. The central vertex has weight $e_{0}$, and replacing this weight with -3 makes the graph rational.

One consequence of the fact that Brieskorn manifolds bound almost rational plumbings is that their Heegaard Floer homology can be calculated combinatorially. In fact Nemethi gives an explicit formula for their (unreduced) tau function, which determines the Heegaard Floer homology, in terms of the Seifert invariants, [13. This formula is in general somewhat difficult to handle in order to say something about Heegaard Floer homology of infinite families of manifolds, a couple of remarkable exceptions being [6, [14] and [24. Instead of attempting to give a closed formula for the Heegaard Floer homology, we are content with a rank calculation.

Proposition 3.3. The ranks of the Heegaard Floer homology of Casson Harer manifolds are as given in Table 2 and Table 3 .

Proof. We can calculate the rank of the Heegaard Floer homology by proceeding as in the proof of Corollary 1.7. Since these manifolds bound both a contractible manifold and an almost rational plumbing, their correction term is zero and their Heegaard Floer homology is supported only in even degrees. Therefore, it suffices to calculate the Casson invariants. In [16], Lemma 1.5, an explicit formula of Casson invariants of Brieskorn manifolds is given. The formula involves certain Dedekind sums, which could be calculated by repeatedly applying the reciprocity law.

One interesting feature of these tables is that the rank of the Heegaard Floer homology increases in the order of $p^{3}$, which is somehow consistent with what we observed in Corollary 1.7 Indeed specializing the case where $p=2 n+1$, and $s=1$, we completely recover Corollary 1.7 from Proposition 3.2. 
TABLE 2 .

\begin{tabular}{|c|c|}
\hline $\begin{array}{c}\text { Brieskorn manifold } \\
\Sigma(p, q, r)\end{array}$ & $\begin{array}{c}\Sigma(p, p s \pm 1, p s \pm 2) \\
p \text { odd }\end{array}$ \\
\hline $\begin{array}{c}\text { Seifert Invariants } \\
\left(e_{0}, p, p^{\prime}, q, q^{\prime}, r, r^{\prime}\right)\end{array}$ & $\left(-1, p, \frac{p-1}{2}, p s \pm 1, s, p s \pm 2, \frac{p s-s \pm 2}{2}\right)$ \\
\hline & $\frac{s\left(p^{2}-1\right)(p s \pm 3)}{24}$ \\
\hline $\begin{array}{c}\text { tau function } \\
\tau(n)\end{array}$ & $\sum_{j=0}^{n-1} 1+j-\left\lceil\frac{j(p-1)}{2 p}\right\rceil-\left\lceil\frac{j s}{p s \pm 1}\right\rceil-\left\lceil\frac{j(p s-s \pm 2)}{2 p s \pm 4}\right\rceil$ \\
$\operatorname{rank}\left(H F^{\operatorname{red}}(-\Sigma)\right)$ & \\
$=-\lambda(\Sigma)$ &
\end{tabular}

TABLE 3 .

\begin{tabular}{|c|c|}
\hline Brieskorn manifold & $\begin{array}{c}\Sigma(p, p s-1, p s+1) \\
p \text { even, } s \text { odd }\end{array}$ \\
\hline $\begin{array}{c}\text { Seifert Invariants } \\
\left(e_{0}, p, p^{\prime}, q, q^{\prime}, r, r^{\prime}\right)\end{array}$ & $\left(-1, p, 1, p s-1, \frac{p s-s-1}{2}, p s+1, \frac{p s-s+1}{2}\right)$ \\
\hline $\begin{array}{c}\operatorname{tau} \text { function } \\
\tau(n)\end{array}$ & $\sum_{j=0}^{n-1} 1+j-\left\lceil\frac{j}{p}\right\rceil-\left\lceil\frac{j(p s-s-1)}{2 p s-2}\right\rceil-\left\lceil\frac{j(p s-s+1)}{2 p s+2}\right\rceil$ \\
\hline $\begin{array}{c}\operatorname{rank}\left(H F^{\mathrm{red}}(-\Sigma)\right) \\
=-\lambda(\Sigma)\end{array}$ & $\frac{p^{3} s^{2}}{24}-\frac{p s^{2}}{24}-\frac{p}{8}$ \\
\hline
\end{tabular}

\section{ACKNOWLEDGMENTS}

The final part of this work was completed while both authors were participating in the FRG Workshop in Miami. They would like to thank N. Saveliev for organizing this workshop. The second author would like to thank R. Gompf for bringing [7] to his attention and P. Ozsváth for an illuminating discussion. A special thanks goes to the anonymous referee who directed us to Maruyama's work [11, [12].

\section{REFERENCES}

[1] Selman Akbulut and Selahi Durusoy, An involution acting nontrivially on Heegaard-Floer homology, Geometry and topology of manifolds, Fields Inst. Commun., vol. 47, Amer. Math. Soc., Providence, RI, 2005, pp. 1-9. MR2189922(2006k:57086)

[2] Proceedings of the Gökova Geometry-Topology Conference 2010, Held in Gökova, May 31June 4, 2010; edited by Selman Akbulut, Denis Auroux and Turgut Önder. International Press, Somerville, MA, 2011. MR2920150 (2012k:57001)

[3] Selman Akbulut and Robion Kirby, Mazur manifolds, Michigan Math. J. 26 (1979), no. 3, 259-284. MR.544597 (80h:57004)

[4] Selman Akbulut and John D. McCarthy, Casson's invariant for oriented homology 3-spheres, An exposition. Mathematical Notes, vol. 36, Princeton University Press, Princeton, NJ, 1990. MR $1030042(90 \mathrm{k}: 57017)$ 
[5] Selman Akbulut and Kouichi Yasui, Corks, plugs and exotic structures, J. Gökova Geom. Topol. GGT 2 (2008), 40-82. MR2466001 (2009k:57036)

[6] M. Borodzik and A. Némethi, Heegaard Floer homologies of $(+1)$ surgeries on torus knots, Acta Math. Hungar. 139 (2013), no. 4, 303-319. MR3061478

[7] Andrew J. Casson and John L. Harer, Some homology lens spaces which bound rational homology balls, Pacific J. Math. 96 (1981), no. 1, 23-36. MR634760 (83h:57013)

[8] E. Harper, On instanton homology of corks $W_{n}$, Topology Appl. 171 (2014), 1-6. MR3207482

[9] Ç. Karakurt, http://www.math.utexas.edu/users/karakurt/.

[10] Yoko Mizuma, On the Casson invariant of homology 3-spheres of Mazur type, Topology Appl. 156 (2009), no. 13, 2292-2294, DOI 10.1016/j.topol.2009.06.003. MR2544138(2010h:57021)

[11] Noriko Maruyama, Notes on homology 3-spheres which bound contractible 4-manifolds. I, J. Tsuda College 13 (1981), 19-31. MR635711 (83c:57003)

[12] Noriko Maruyama, Notes on homology 3-spheres which bound contractible 4-manifolds. II, J. Tsuda College 14 (1982), 7-24. MR662274 (83j:57007)

[13] András Némethi, On the Ozsváth-Szabó invariant of negative definite plumbed 3-manifolds, Geom. Topol. 9 (2005), 991-1042, DOI 10.2140/gt.2005.9.991. MR2140997 (2006c:57011)

[14] András Némethi, On the Heegaard Floer homology of $S_{-d}^{3}(K)$ and unicuspidal rational plane curves, Geometry and topology of manifolds, Fields Inst. Commun., vol. 47, Amer. Math. Soc., Providence, RI, 2005, pp. 219-234. MR2189934 (2007g:57026)

[15] András Némethi and Liviu I. Nicolaescu, Seiberg-Witten invariants and surface singularities, Geom. Topol. 6 (2002), 269-328 (electronic), DOI 10.2140/gt.2002.6.269. MR.1914570 (2003i:14048)

[16] Walter Neumann and Jonathan Wahl, Casson invariant of links of singularities, Comment. Math. Helv. 65 (1990), no. 1, 58-78, DOI 10.1007/BF02566593. MR1036128 (91c:57022)

[17] Peter Ozsváth and Zoltán Szabó, Holomorphic disks and topological invariants for closed three-manifolds, Ann. of Math. (2) 159 (2004), no. 3, 1027-1158, DOI 10.4007/annals.2004.159.1027. MR.2113019(2006b:57016)

[18] Peter Ozsváth and Zoltán Szabó, Holomorphic disks and three-manifold invariants: properties and applications, Ann. of Math. (2) 159 (2004), no. 3, 1159-1245, DOI 10.4007/annals.2004.159.1159. MR2113020 (2006b:57017)

[19] Peter Ozsváth and Zoltán Szabó, Absolutely graded Floer homologies and intersection forms for four-manifolds with boundary, Adv. Math. 173 (2003), no. 2, 179-261, DOI 10.1016/S0001-8708(02)00030-0. MR1957829 (2003m:57066)

[20] Peter Ozsváth and Zoltán Szabó, Holomorphic triangles and invariants for smooth four-manifolds, Adv. Math. 202 (2006), no. 2, 326-400, DOI 10.1016/j.aim.2005.03.014. MR2222356 (2007i:57029)

[21] Peter Ozsváth and Zoltán Szabó, Holomorphic triangle invariants and the topology of symplectic four-manifolds, Duke Math. J. 121 (2004), no. 1, 1-34, DOI 10.1215/S0012-7094-0412111-6. MR 2031164(2004m:57059)

[22] Peter Ozsváth and Zoltán Szabó, On the Floer homology of plumbed three-manifolds, Geom. Topol. 7 (2003), 185-224 (electronic), DOI 10.2140/gt.2003.7.185. MR.1988284(2004h:57039)

[23] Nikolai Saveliev, A note on Akbulut corks, Math. Res. Lett. 10 (2003), no. 5-6, 777-785. MR2024733 (2004i:57037)

[24] E. Tweedy, Heegaard Floer homology and several families of Brieskorn spheres, Topology Appl. 160 (2013), no. 4, 620-632. MR.3018077

Department of Mathematics, Michigan State University, East Lansing, Michigan 48824

E-mail address: akbulut@math.msu.edu

Department of Mathematics, The University of Texas at Austin, 2515 Speedway, Stop C1200, Austin, Texas 78712

E-mail address: karakurt@math.utexas.edu 